\title{
Non-invasive positive pressure ventilation in acute hypercapnic respiratory failure: clinical experience of a respiratory ward
}

\author{
R. Scala, M. Naldi, I. Archinucci, G. Coniglio
}

\begin{abstract}
Non-invasive positive pressure ventilation in acute hypercapnic respiratory failure: clinical experience of a respiratory ward. R. Scala, M. Naldi, I. Archinucci, G. Coniglio.

Background: Although a controlled trial demonstrated that non-invasive positive pressure ventilation (NIV) can be successfully applied to a respiratory ward (RW) for selected cases of acute hypercapnic respiratory failure (AHRF), clinical practice data about NIV use in this setting are limited. The aim of this observational study is to assess the feasibility and efficacy of NIV applied to AHRF in a RW in everyday practice.

Methods: Twenty-two percent (216/984) of patients consecutively admitted for AHRF to our RW in Arezzo (years: 1996-2003) received NIV in addition to standard therapy, according to pre-defined routinely used criteria. Tolerance, effects upon arterial blood gases (ABG), success rate (avoidance a priori criteria for intubation) and predictors of failure of NIV were analysed.
\end{abstract}

\begin{abstract}
Results: Nine patients $(4.2 \%)$ were found to be intolerant to NIV, while the remaining 207 (M: 157, F: 50; mean (SD) age: 73.2 (8.9) yrs; COPD: $71.5 \%$ ) were ventilated for $>1$ hour. ABG significantly improved after two hours of NIV (pH: 7.32 (0.06) versus median (Interquartiles) 7.28 (7.24-7.31), p<0.0001; $\mathrm{PaCO}_{2}$ : 71.9 (13.5) $\mathrm{mmHg}$ versus 80.0 (15.2) $\mathrm{mmHg}, \mathrm{p}<0.0001 ; \mathrm{PaO}_{2} / \mathrm{FiO}_{2}: 212$ (66) versus $184(150-221), p<0.0001)$. NIV succeeded in avoiding intubation in 169/207 patients $(81.6 \%)$ with hospital mortality of $15.5 \%$. NIV failure was independently predicted by Activity of Daily Living score, pneumonia as cause of AHRF and Acute Physiology and Chronic Health Evaluation III score.

Conclusions: In clinical practice NIV is feasible, effective in improving ABG and useful in avoiding intubation in most AHRF episodes that do not respond to the standard therapy managed in an RW adequately trained in NIV. Monaldi Arch Chest Dis 2004; 61: 2, 94-101.
\end{abstract}

Keywords: Non-invasive positive pressure ventilation, acute respiratory failure, respiratory ward, endotracheal intubation, hospital mortality, COPD, co-morbidity.

Unità Operativa di Pneumologia, ASL8, Ospedale S. Donato, Arezzo (Italy).

Correspondence: Dr Raffaele Scala; Via Caduti sul Lavoro, 8; 52100 Arezzo (Italy); e-mail: raffaele_scala@hotmail.com

\section{Introduction}

Prospective randomised controlled studies have shown that NIV added to standard treatment reduces the need for endotracheal intubation (ETI), hospital mortality and length of stay in selected patients with acute hypercapnic respiratory failure (AHRF), mostly due to chronic obstructive pulmonary disease (COPD), compared to standard treatment. This benefit has been proved not only when NIV was provided in intensive care settings but, recently, also in respiratory ward (RW) [1-5].

However, the field of application of NIV in clinical practice is not completely defined as the results of well designed randomised studies may differ from those obtained in clinical routine for several reasons [6, 7]. Firstly, comprehensive logs were often not kept of excluded patients in interventional trials $[2,5]$ and, consequently, did not clarify the role of NIV in "the real world"; in the study of Brochard et al. [3], only $31 \%$ of the screened COPD patients were recruited for NIV. Secondly, stringent selection criteria adopted by the randomised trials may exclude subsets of patients who could take advantage of NIV (i.e. advanced age, reduced level of consciousness, endstage lung, not-to be intubated patients) $[8,9]$. Thirdly, in concealed but not fully double blinded prospective trials greater attention was generally focused on assuring the success of NIV. As recently underlined by the British Thoracic Society, conclusions about the indications for NIV from the evidence available must be made with caution [7].

Large scale data about the everyday use of NIV are referred to intensive care unit (ICU) and respiratory high dependency unit (RHDU), where NIV was used in 17-50\% of AHRF episodes [10-13].

The perspective of a wider, even if "safe", routine of NIV in a RW for selected episodes of AHRF is extremely appealing due to the growing gap between the evidence-based increase in the demand for NIV and the limited resources allocated for ICU and RHDU in many countries $[14,15]$. In $\mathrm{RW}$, NIV is generally recommended to be started in the early phase of AHRF for less severe acidosis $(\mathrm{pH}>7.30)$ and to be continued if clinical sta- 
tus and blood gases improved after a 1-2 hours [6, 7]. However, data concerning non-study use of RW are limited [16-19]. Observational studies, when applied to proper design and methods, may be very helpful in clarifying the "real world" therapeutic effectiveness of a specific technique, such as NIV, in a specific setting once it has already been validated by controlled trials.

The purpose of this observational study is to investigate the feasibility and the efficacy of NIV for the treatment of AHRF in clinical practice in an RW adequately trained in this ventilatory technique.

\section{Methods}

This observational study was performed in a 25 bed RW in "S. Donato" Hospital of Arezzo between January 1996 and January 2003. The study protocol was approved by the local Ethical Committee of our hospital; the informed consent was obtained in all cases from either the patient or their next kin.

\section{Patient selection}

All patients with different underlying diseases consecutively admitted to our RW for an episode of AHRF $\left(\mathrm{PaCO}_{2}>45 \mathrm{mmHg}\right.$ and $\left.\mathrm{PaO}_{2} / \mathrm{FiO}_{2}<250\right)$ were considered for the study. They were enrolled if, despite the controlled oxygen therapy through a Venturi mask at the minimum $\mathrm{FiO}_{2}$ able to achieve a transcutaneous oxygen saturation $\left[\mathrm{SpO}_{2}\right] \geq 90 \%$ and the appropriate medical treatment (i.e. salbutamol and ipratropium bromide via aerosol every four hours, intravenous aminophylline, corticosteroids, antibiotics, cardiovascular drugs and therapy for coexistent comorbidities if necessary) fulfilled all the following criteria: 1) $\mathrm{pH}<7.33$ or $\mathrm{Pa}-$ $\mathrm{CO}_{2}>55 \mathrm{mmHg}$; 2) $\mathrm{PaO}_{2} / \mathrm{FiO}_{2}<250$; 3) dyspnoea at rest with respiratory rate $(\mathrm{RR})>25$ breaths/min; 4) use of accessory respiratory muscles or paradoxical abdominal breathing.

Exclusion criteria for NIV were: a) facial deformity sufficient to affect mask fitting; b) severe encephalopathy unrelated to hypoxemia and/or hypercapnia; c) overt gastro-intestinal bleeding; d) acute ischaemic heart disease; e) need for urgent ETI due to cardiac or respiratory arrest, prolonged respiratory pauses and psychomotor agitation requiring sedation [3].

\section{Non-invasive ventilation}

All patients enrolled in the study were managed in our RW by nurses and physicians adequately trained in NIV technique. Nurses had attended a 10 hour training course on NIV and monitoring of acute respiratory patients in the two preceding months; they were kept up to date by means of a yearly 4 hour course. Mean nurse:patient ratio was 1:9. During the earliest phase of NIV one pulmonologist was continuously present at the patient's bedside; later the patient was closely looked after by one nurse with one physician available on call. Electrocardiogram, $\mathrm{SpO}_{2}$ and non-invasive blood pressure were monitored continuously. ETI and transfer to ICU was promptly available in case of NIV failure.

NIV was delivered by means of Bi-PAP Vision (Respironics, Inc., Pittsburgh, PA, USA) in S/T mode via a well fitting either nasal or facial mask (Mirage, ResMed, Norh Ride, Australia), depending on the amount of mouth air leaks and on the level of co-operation. A Plateau exhalation valve was applied to the circuit in order to minimize $\mathrm{CO}_{2}$-rebreathing [20]; IPAP was initially set at 10 $\mathrm{cmH}_{2} \mathrm{O}$ and then gradually increased to a maximum of $25 \mathrm{cmH}_{2} \mathrm{O}$ depending on clinical and blood gas response as well as on the patient's tolerance. EPAP was always set at $5 \mathrm{cmH}_{2} \mathrm{O}$ and backup RR at 16 breath/min. $\mathrm{FiO}_{2}$ was adjusted in order to keep $\mathrm{SpO}_{2} \geq 90 \%$. Patients were encouraged to use NIV for as long as possible, aiming for at least 6-8 hours per day split into three sessions; NIV was discontinued when all the following criteria was retained for $>24$ hours while breathing oxygen: $\mathrm{pH}>7.35 ; \mathrm{PaO}_{2} / \mathrm{FiO}_{2}>250 ; \mathrm{RR}<25$ breaths/min.

\section{Criteria for endotracheal intubation}

NIV was considered to have failed if at least one of the following criteria for ETI [3] was satisfied: 1) cardiac arrest or severe haemodynamic instability (systolic blood pressure $<80 \mathrm{mmHg}$ ) with loss of alertness; 2) respiratory arrest or gasping or prolonged respiratory pauses; 3 ) psychomotor agitation requiring sedation; 4) severe difficulty in clearing bronchial secretions; 5) worsening of gas exchange (at least two of the following: $\mathrm{pH}<7.30$ and $<0.02$ with respect to baseline; $\mathrm{PaO}_{2} / \mathrm{FiO}_{2}<150$ and $<20$ with respect to baseline; $\mathrm{PaCO}_{2}>20 \%$ with respect to baseline) and/or level of consciousness (Kelly score $>1$ with respect to baseline) [21] during NIV for at least 6 hours with the best ventilator setting; 6) inability to tolerate the mask because of discomfort.

In the case of refusal of invasive ventilation by the patient and/or their family NIV failure was considered when the a priori criteria for ETI were met.

\section{Measurements}

The following variables were collected:

Demographic, clinical and functional data when stable: (a) age and sex; (b) underlying disease: COPD, chest wall deformity (CWD), chronic heart failure (CHF), neuromuscular disorders (NM), obesity-hypoventilation syndrome (OHS); (c) spirometric data within six months from admission: forced expiratory volume in one second $\left(\mathrm{FEV}_{1}\right)$, forced vital capacity (FVC); (d) body mass index (BMI) $\left(\mathrm{Kg} / \mathrm{m}^{2}\right)$; (e) functional limitation evaluated with Activities of Daily Living (ADL) score [22]; (f) use of long-term oxygen therapy (LTOT) or home mechanical ventilation (HMV); (g) number of comorbidities [23];

Clinical and functional data at admission: (a) sources of patients: emergency department (ED), ICU, medical ward, pulmonologist ambulatory, nursing home; (b) causes of AHRF: upper airway 
infections, community-acquired pneumonia, pulmonary embolism, acute heart decompensation, others [24]; (c) acute severity of illness assessed by Acute Physiology and Chronic Health Evaluation (Apache III) score [25]; (d) level of consciousness measured with Kelly score [21].

Data about NIV: (a) arterial blood gases (ABG) at the admission in RW, before starting NIV (pre-NIV) and after 2 hours of NIV (2h-NIV); (b) level of IPAP; (c) interface; (d) tolerance assessed by a simplified score (1=low, 2=medium, 3=good) [24]; (e) NIV-related complications.

\section{Statistical Analysis}

The Kolmogorov-Smirnov test was used to test whether all recorded variables were normally distributed ( $>>0.05)$. Continuous data is expressed as mean (standard deviation (SD)) or as median (interquartile range (IQR)) if distributed respectively normally or not; categorical data are presented as frequency $(\%)$.

Comparisons between ABG values measured at different times were done with two tailed paired $\mathrm{t}$ test (parametric data) and Wilcoxon test (nonparametric data).

To identify the variables correlated with NIV outcome, univariate analysis was firstly performed between patients successfully an unsuccessfully treated with NIV. Continuous variables were compared with two-tailed unpaired test (parametric data) or Mann-Whitney U test (non-parametric data). Categorical data were compared using Chisquare test or, when appropriate, Fisher's exact test. A p value $<0.05$ was considered statistically significant. Due to the limited number of variables that may be entered in the multivariate analysis (one for every 10 events) stringent selection criteria were employed [26]. Variables with $p$ value $<0.01$ on univariate analysis were considered eli- gible for multivariate analysis. To avoid multicollinearity, we looked for correlations between all eligible variables with Spearman's test (significance: $\mathrm{p}<0.01$ ): if highly correlated variables were found, these which are likely to be dependent on the others were excluded. Then, all selected variables were entered into a stepwise forward logistic regression model (entry criteria: $\mathrm{p}<0.05$ ); the variables with $\mathrm{p}$ value $<0.05$ in this model were considered statistically significant and their predictive value was reported as the adjusted odds ratio (OR) with $95 \%$ confidence interval (CI).

Analyses were performed using the version 10.0 of the SPSS statistical software package (SPSS, Inc., Chicago Illinois).

\section{Results}

During the study period 984 consecutive patients with AHRF (pH: 7.38 (0.05); $\mathrm{PaCO}_{2}: 50.5$ (12.5) $\mathrm{mmHg} ; \mathrm{PaO}_{2} / \mathrm{FiO}_{2}: 235$ (41)) of different aetiologies were admitted to our RW in Arezzo. As $73.5 . \%$ (723/984) improved quickly with standard therapy and $4.5 \%$ (45/984) needed urgent ETI within 1 hour from the admission, 22\% (216/984) entered in the study. The number/year of AHRF treated with NIV was 27 (4.6) (range: 19-33).

Enrolled patients received NIV 11.4 (2-63.75) hours after the entrance into RW as, despite standard therapy, respiratory acidosis significantly worsened (pH: 7.28 (7.24-7.31) versus 7.32 (0.06), $\mathrm{p}<0.0001 ; \mathrm{PaCO}_{2}: 80.0$ (15.2) mmHg versus 69.4 (14.2) $\mathrm{mmHg}, \mathrm{p}<0.0001)$ with a non significant drop in oxygenation status $\left(\mathrm{PaO}_{2} / \mathrm{FiO}_{2}\right.$ : 184 (150221 ) versus $200(58), p=0.157)$. Nine out of the 216 patients recruited $(4.2 \%)$ did not tolerate NIV, while the remaining 207/216 patients were ventilated for $>1$ hour (figure 1). The most common underlying disease was COPD $(71.5 \%)$ (figure 2$)$; the main source was ED (56\%) (figure 3 ). In 6 cases

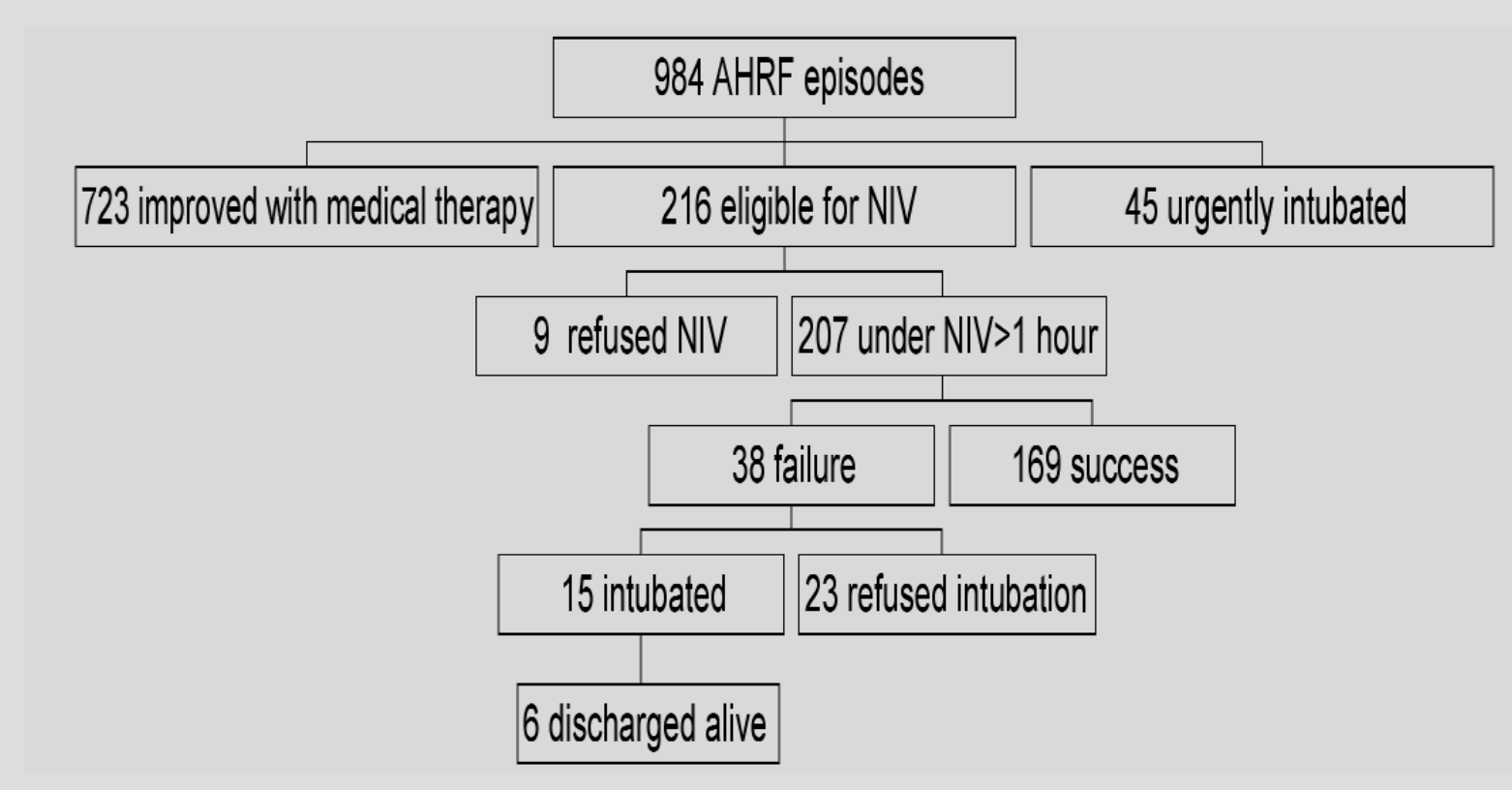

Fig. 1. - Distribution of the patients according to treatment and response to NIV.

NIV: non-invasive positive pressure ventilation; AHRF: acute hypercapnic respiratory failure. 


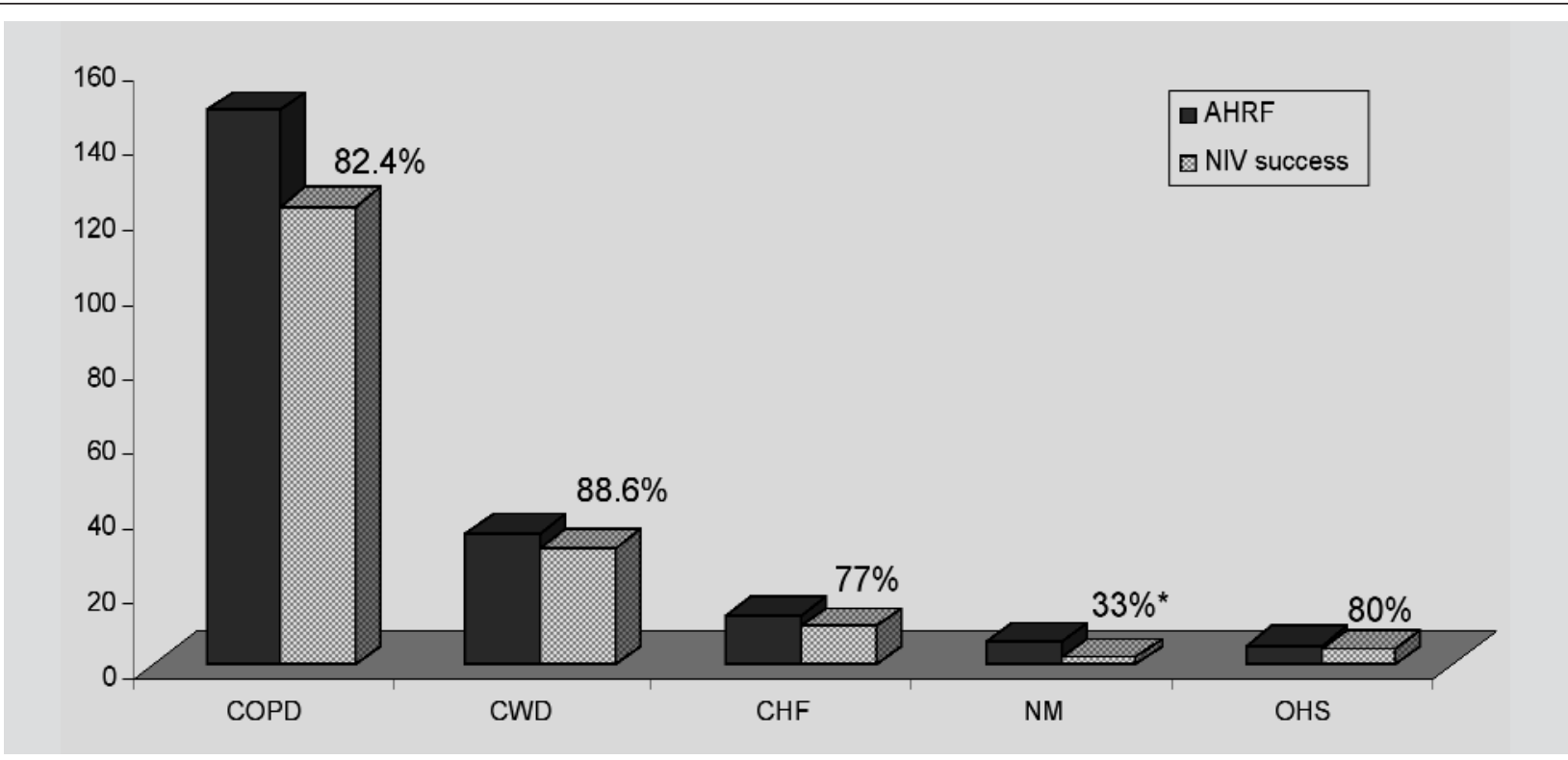

Fig. 2. - Typology of underlying diseases in the studied population ( $\mathrm{n}=207$ patients): COPD (71.5\%), CWD (16.9\%), CHF (6.3\%), NM (2.9\%), OHS (2.4\%) NIV: non-invasive positive pressure ventilation; AHRF: acute hypercapnic respiratory failure; COPD: chronic obstructive pulmonary disease; CWD: chest wall deformities; CHF: chronic heart failure; NM: neuromuscular disorders; OHS: obesity-hypoventilation syndrome. $* \mathrm{p}<0.05$ versus other types of diseases.

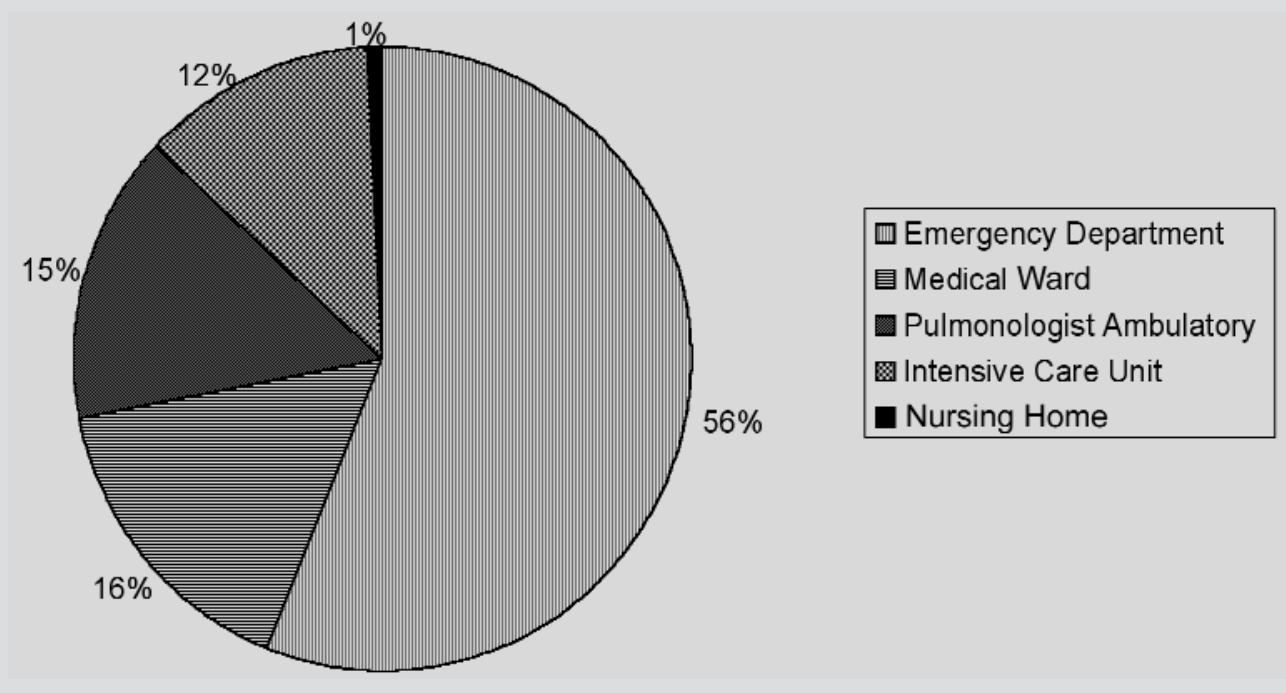

Fig. 3. - Sources of the studied population ( $n=207$ patients)

coming from ICU, NIV was used to treat post-extubation AHRF. Tables 1 and 2 showed respectively "historic" and "acute" data of the studied population. Twenty percent of patients (42/207) were more than 75 years-old while $56 \%$ of them $(116 / 207)$ showed at least one co-morbidity, mainly cardiovascular. Alertness was altered (Kelly score $>1)$ in $46.4 \%$ of cases $(96 / 207)$. ABG significantly improved after 2 hours of NIV.

NIV was delivered for a total of 41 (22-73) hours (1 st day: 8.5 (6-13.5) hours) over 6 (3-10) days. NIV was well tolerated with a compliance score $>1$ in $59 \%$ of patients. IPAP applied was 18 (14.5-20) $\mathrm{cmH}_{2} \mathrm{O}$. Nasal masks were used in $51.7 \%$ of cases $(n=107)$, facial masks in $48.3 \%$ of cases $(n=100)$. NIV-related complications were observed in $30.6 \%$ of patients (63/207): nasal lesions $(n=37 ; 18 \%)$, abdominal distension $(n=24$; $11.6 \%)$, conjunctivitis $(n=11 ; 5.3 \%)$; all of them were reversible.

NIV succeeded in avoiding ETI in 169/207 patients $(81.6 \%)$. The success rate was significantly lower in NM compared with other disorders (figure 2). Figure 4 showed causes and timing of failure. NIV failed after 3 (1.75-8) days with $58 \%$ of cases needing ETI within the first 48 hours; "late failure" after an initial improvement [24] occurred in $12.5 \%$ of cases ventilated for $>48$ hours (22/176). Worsening of ABG and/or Kelly score was the main cause of failure in all types of diseases except for NM in whom ETI was mostly needed ( $75 \%$ of cases) for retention of secretions. 
Table 1. - Demographic, clinical and functional data when stable before the admission in our respiratory ward ( $n=207$ patients)

\begin{tabular}{ll}
\hline Age yrs & $73.2(8.9)$ \\
Male n (\%) & $157(75.8)$ \\
FEV $_{1} \%$ predicted median (IQR)* & $29(23-39)$ \\
FVC\% predicted* & $60(17.6)$ \\
BMI Kg/m² median (IQR) & $24.8(21.6-28.0)$ \\
ADL score <2 n (\%) & $42(20.3)$ \\
Comorbidities $\geq 1 \mathrm{n}(\%)$ & $116(56)$ \\
LTOT n (\%) & $129(62.3)$ \\
HMV n (\%) & $15(7.2)$ \\
\hline
\end{tabular}

Data are expressed as mean (standard deviation) unless otherwise stated.

$\mathrm{IQR}$ : interquartiles; $\mathrm{FEV}_{1}$ : forced expiratory volume in one second; FVC: forced vital capacity; BMI: body mass index; ADL: activities of daily living; LTOT: long-term oxygen therapy; HMV: home mechanical ventilation.

* available in 156 patients.

Intolerance to keep on NIV led to failure in $6 / 38$ cases $(15.8 \%)$. Only 15 subjects were intubated as in the others ETI was refused. Nine intubated patients died in ICU after 23 (1.5-32.5) days for septic shock (2 cases), multiple organ dysfunction syndrome ( 3 case), cardiac arrest (1 case), massive pulmonary embolism ( 1 case), withdrawal of ventilation due to wishes of patients and their familiars (2 cases of amyotrophic lateral sclerosis). Among the 6 intubated patients who survived, two (COPD and Duchenne muscular dystrophy) were discharged on HMV via tracheostomy; one COPD patient was successfully weaned through NIV. Inhospital mortality of the enrolled population was
Table 2. - Clinical and physiologic data during the stay in our respiratory ward ( $n=207$ patients)

\begin{tabular}{ll}
\hline Causes of AHRF & $124(59.9)$ \\
$\quad$ Upper airway infection n (\%) & $31(15)$ \\
Pneumonia n (\%) & $39(18.8)$ \\
Acute heart decompensation n (\%) & $7(3.4)$ \\
Pulmonary embolism n (\%) & $6(2.9)$ \\
Others n (\%) & $59(50-72)$ \\
Apache III score median (IQR) & $96(46.4)$ \\
Kelly score> 1 n (\%) & $7.28(7.24-7.31)$ \\
$\mathrm{pH}$ pre-NIV * median (IQR) & $184(150-221)$ \\
$\mathrm{PaO}_{2} / \mathrm{FiO}_{2}$ pre-NIV * median (IQR) & $80(15.2)$ \\
$\mathrm{PaCO}_{2}$ pre-NIV mmHg* & $7.32(0.06)$ \\
$\mathrm{pH}_{2} \mathrm{~h}-\mathrm{NIV}^{\wedge}$ & $212(66)$ \\
$\mathrm{PaO}_{2} / \mathrm{FiO}_{2} 2 \mathrm{~h}-\mathrm{NIV}^{\wedge}$ & $71.9(13.5)$ \\
$\mathrm{PaCO}_{2} 2 \mathrm{~h}-\mathrm{NIV}$ mmHg^ &
\end{tabular}

Data are expressed as mean (standard deviation) unless otherwise stated.

IQR: interquartiles; AHRF: acute hypercapnic respiratory failure; NIV: non-invasive positive pressure ventilation; Apache III: acute physiology and chronic health evaluation. $*$ while breathing $\mathrm{O}_{2}$ at the needed concentration.

$\wedge \mathrm{p}<0.0001$ versus pre-NIV.

$15.5 \%$ (32/207). At the discharge LTOT and HMV were prescribed respectively in 25 and 28 patients. The length of hospital stay of whole population was 15 (10-21) days.

Among the 12 variables significantly associated with NIV failure on univariate analysis, seven of them $(p<0.01)$ were eligible for entry in the multivariate analysis. Due to the significant correlations found between pneumonia and hours of NIV (first day) $(r=0.185 ; p<0.001)$, Kelly score and Apache III score $(\mathrm{r}=0.721 ; \mathrm{p}<0.001)$,

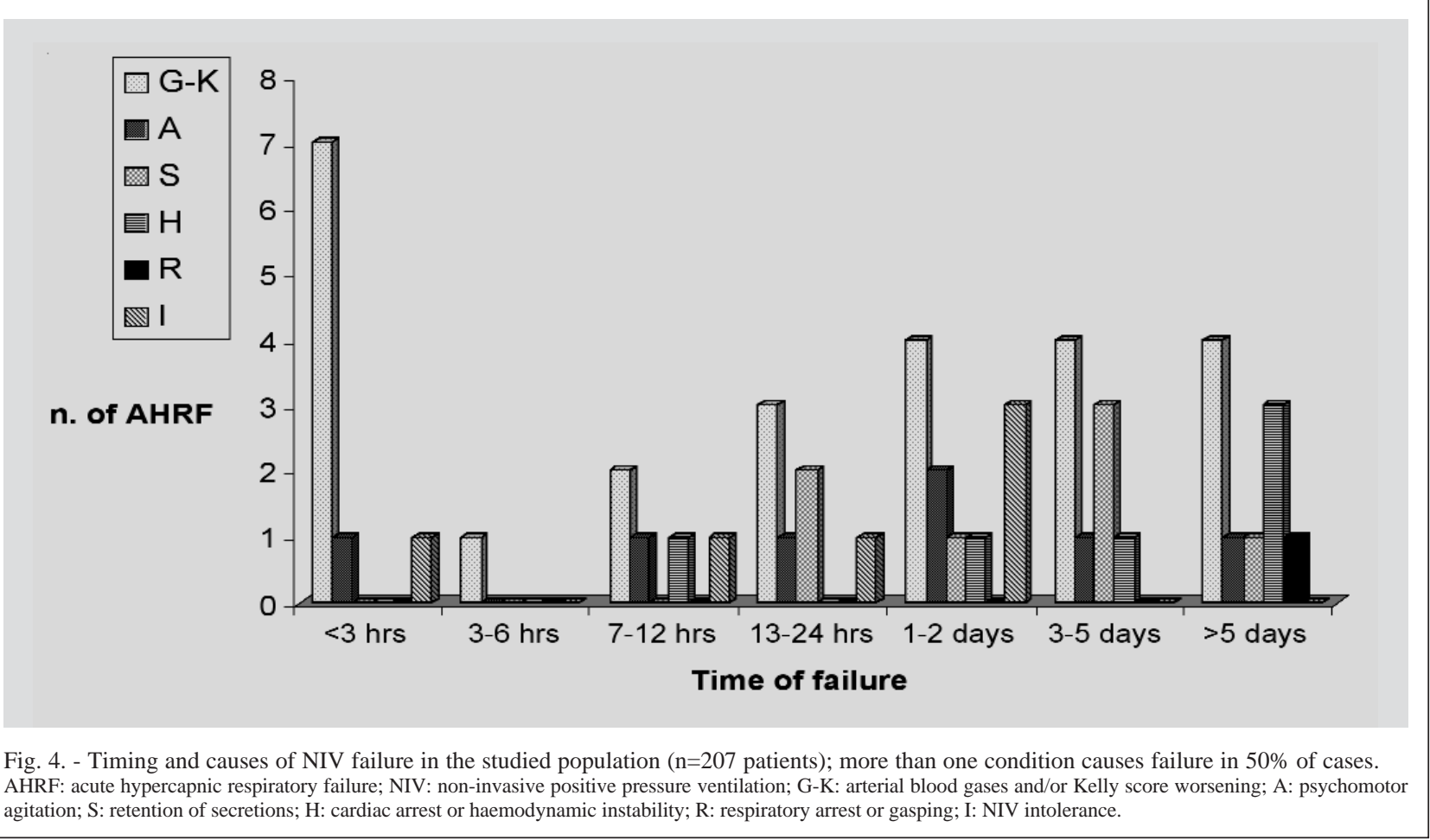


$\mathrm{PaO}_{2} / \mathrm{FiO}_{2}$ (2h-NIV) and Apache III score ( $\mathrm{r}=$ $-0.293 ; p<0.001$ ), four variables were selected to be entered in the stepwise logistic regression model. The multivariate analysis showed that NIV failure was independently predicted by ADL score, pneumonia as cause of AHRF and Apache III score (table 3 ). The same three variables resulted independently associated with NIV failure in COPD patients according to multivariate analysis.

\section{Discussion}

The main finding of our study is that in everyday clinical practice of a RW NIV is feasible and useful in preventing intubation in most treated patients with AHRF, whose predominant aetiology is COPD. In our experience, only $4.2 \%$ of patients who received NIV were not able to cope with the mask due to discomfort. In tolerant patients, NIV improved significantly gas exchange after 2 hours and succeeded in avoiding ETI and consequent transfer to ICU in $81.6 \%$ of the cases. Poorer pre-admission functional activity (as assessed by ADL score), greater severity of acute illness (measured with Apache III score) and pneumonia, as cause of AHRF, were independently associated with NIV failure.

Although a large controlled trial demonstrated that NIV can be set up and successfully used in a RW for COPD exacerbations with mild acidosis, clinical practice data about RW-based NIV use in unselected AHRF cases are limited [16-19]. In UK, where there is shortage of ICU/RHDU beds, NIV is performed exclusively on respiratory or general ward in $40 \%$ of cases [19]. A recent European survey [15] showed that $20 \%$ of RHDU (i.e. Respiratory Monitoring Unit), whose predominant activity is NIV, provided a low level of care which is similar to that offered by RW.
In the observational study conducted on our RW in a series of 207 unselected non-invasively ventilated patients, $20 \%$ of them were over 75 years-old, $56 \%$ had at least one co-morbidity and $46 \%$ showed hypercapnic-related neurologic alterations. Severe comorbidities and impaired consciousness are described as "classical" contraindications to NIV since they were exclusion criteria for the controlled trials $[6,7]$. However, boundaries for the use of NIV are not always well defined as they are continuing in expansion. It's certainly clear that ETI and conventional ventilation remain the "gold standard" for AHRF in patients with acute multiple organ failure and/or coma with inability to protect airway and/or uncontrolled confusion and agitation. In the opposite, rather than contraindicated NIV is not proven in AHRF with compensated multiple comorbidities and/or milder degree of hypercapnic encephalopathy. Consequently, in these circumstances the choice of NIV should be driven by the individual evaluation of the case in the clinical ground; therefore, a trial of NIV could be justified if ETI is not considered appropriate or refused [7]. In agreement with our finding, encouraging results were reported in selected patients who were either elderly or refused ETI or showed signs of $\mathrm{CO}_{2}$-related encephalopathy $[8,9]$.

With regards to the needs of NIV in the clinical practice, we found that $22 \%$ of patients who entered in our RW for AHRF thereafter required NIV for deterioration of respiratory conditions despite optimised medical therapy and controlled oxygen therapy. As a matter of fact, NIV was applied every year to a mean of 27 AHRF cases, mostly due to COPD decompensations and coming from ED. In our Hospital, which serves a population of about 90.000 inhabitants, RW is the unique

Table 3. - Variables predicting NIV failure ( $\mathrm{n}=207$ patients)

\begin{tabular}{|c|c|c|c|c|}
\hline \multirow[b]{2}{*}{ Variable } & \multirow{2}{*}{$\begin{array}{l}\text { Univariate analysis } \\
\qquad p \text { value }\end{array}$} & \multicolumn{3}{|c|}{ Multivariate analysis } \\
\hline & & Adjusted OR & $95 \% C I$ & $p$ value \\
\hline ADL score* & $<0.001$ & 0.166 & $0.800-0.347$ & $<0.001$ \\
\hline Apache III score* & $<0.001$ & 1.032 & $1.010-1.055$ & 0.004 \\
\hline Days of NIV* & $<0.001$ & & & \\
\hline Kelly score & $<0.001$ & & & \\
\hline Hours of NIV (first day) & 0.001 & & & \\
\hline $\mathrm{PaO}_{2} / \mathrm{FiO}_{2}$ 2h-NIV & 0.002 & & & \\
\hline Pneumonia* & 0.004 & 3.922 & $1.400-10.982$ & 0.009 \\
\hline BMI Kg/m² & 0.012 & & & \\
\hline pH 2h-NIV & 0.013 & & & \\
\hline $\mathrm{PaO}_{2} / \mathrm{FiO}_{2}$ pre-NIV & 0.014 & & & \\
\hline Face mask & 0.017 & & & \\
\hline Comorbidities & 0.028 & & & \\
\hline
\end{tabular}

* variables selected to be entered in multivariate analysis.

OR: odds ratio; CI: confidence interval.

NIV: non-invasive positive pressure ventilation; ADL: activities of daily living; Apache III: acute physiology and chronic health evaluation; BMI: body mass index 
environment where NIV is performed; for this reason, sources of our patients were ICU, general ward and pneumology clinic, as well as ED. In a one-year prospective prevalence study conducted in a typical UK hospital serving a population of 250.000 , Plant et al. [27] showed that $35 \%$ of patients admitted to ED for an hypercapnic exacerbation of COPD met the criteria for NIV once entered in RW. The authors estimated that 72 COPD exacerbations every year needed to be treated with NIV in a RW for persistent acidosis $(\mathrm{pH}<7.35)$ despite controlled oxygen therapy in ED. Nevertheless, these results are difficult to compare with our data because of differences in: time of observation, provision of care in ED, ICU beds availability, age and underlying diseases of the population, causes and sources of AHRF.

In our study NIV was well accepted as it was used for more than one hour by $95.8 \%$ of the patients, a rate slightly higher than that reported in other RW studies $(87 \%, 93 \%)[1,5]$.

The ability of quickly improving $\mathrm{ABG}$, as described by other authors $[1-3,5,18]$, was due to the known physiologic effects of NIV on respiratory muscles and alveolar ventilation [28].

The majority of the patients with AHRF who received NIV in our study were affected by COPD, in whom controlled trials demonstrated the greater benefits of acute NIV [6, 7].

The success of NIV to avoid the need of ETI in about $80 \%$ of our patients was in agreement with data of the literature $(50-93 \%)[6,7]$. Specifically, NIV success and hospital mortality in COPD were similar to those reported by Plant et al. [5] in the NIV arm $(82.4 \%$ versus $85 \% ; 14.9 \%$ versus $15 \%$, respectively) of their controlled trial in RW even though acidosis was milder in the latter (median (IQR) 7.32 (7.25-7.35) versus 7.28 (7.25-7.31)). This finding seems to be surprising as in AHRF with $\mathrm{pH}<7.30$ Plant et al. [5] did not show any significant advantages of NIV compared to standard therapy. However, in the multicenter UK trial, most of the wards involved had not had any previous experience of NIV, which was managed by nurses according to a simple protocol with a simple ventilator. In our experience, the nurses' training in the two months preceding NIV implementation, the presence of one physician in the earliest phase of AHRF and the use of a more sophisticated ventilator could at least partially explain the similar percentage of success obtained in more acidotic patients.

Rate of failure was high in six neuromuscular patients $(77 \%), 5$ of them affected by amyotrophic lateral sclerosis. In this disorder prognosis is poor and NIV is less effective and fails especially for difficulty in removing secretions [29], as happened in our study.

It's known that, even after an initial improvement, NIV "lately fails" in 15-28\% of the cases with an high in-hospital mortality $(22-68 \%)$ [3, 24]; accordingly, "late" failure of NIV occurred in $12.5 \%$ of our patients who showed a mortality of $54.5 \%$.

Among several parameters significantly associated with NIV failure in the present study, three of them were independent predictors of outcome in multivariate analysis (ADL score, Apache III score, pneumonia). Accordingly, it's has been described the prognostic value of pre-morbid activity [24], illness severity [30, 31] and pneumonia [31] in AHRF requiring NIV.

It has been reported that $[32,33]$ NIV is less likely to success in "lung failure" triggered by community-acquired pneumonia than in "pump failure", due to its main physio-pathologic effect of unloading respiratory muscles as well as the difficulty in clearing secretions during non-invasive support. In a randomised controlled study, Confalonieri et al. [34] found that NIV reduced the need of ETI in the subset of acute hypercapnic and not of hypoxemic patients with severe pneumonia. In our study, at baseline $\mathrm{PaO}_{2} / \mathrm{FiO}_{2}$ was significantly lower in patients with $(n=31)$ than without pneumonia $(n=176)$ (168 (43) versus 194 (63); $\mathrm{p}=0.005)$ without significant differences in $\mathrm{PaCO}_{2}$ (76.9 (14.1) versus 80.5 (15.4); $\mathrm{p}=0.225)$ and $\mathrm{pH}$ (7.27 (0.06) versus $7.27(0.06) ; \mathrm{p}=0.843)$.

Median use of ventilator in the first day of NIV in COPD patients was not different from Plant et al. study (8 hours) [5]; differently, we reported a longer length of NIV (median 6 vs 3 days) and of hospital stay (15 vs 10 days) probably for the greater severity of our cases.

Some limitations of this study must be underlined. Firstly, the long period of observation ( 8 years) may have introduced a bias. Although ventilator, masks and nursing remained unchanged over the time, a "learning effect" could have affected the results of our study. In fact, as shown in a RHDU experience [35], the rate of success of NIV that we reported in the last four years was similar to that obtained in the first four years, despite a trends towards a greater severity of the patients ventilated in the former (in terms of ADL, Kelly score, $\mathrm{pH}$ and $\mathrm{PaCO}_{2}$ ). Secondly, although we employed objective criteria for the use of NIV, the results of this uncontrolled study are based on the assumption that all recruited patients really needed a ventilatory support; similar results could have been reached at least in some cases with the sole standard therapy. However, the significant impairment of acidosis with standard therapy before NIV institution should have minimised this bias.

In conclusion, according to this study, NIV may be considered a feasible and effective technique of ventilatory support in clinical practice within a RW as it may prevent ETI in most of patients with AHRF non responsive to standard therapy. Our experience confirmed that implementation of NIV in a RW requires an adequate training of nurse's staff and the prompt availability of ETI in case of failure. Furthermore, it should be always kept in mind that management of more severe cases of AHRF which are more likely to fail is strongly recommended in a setting with higher levels of care, unless ETI is questionable or refused.

Acknowledgement: We are very grateful to all nursing and physiotherapist staff of our Respiratory Ward for their invaluable help in supporting NIV use in our acute respiratory patients throughout these difficult years. 


\section{References}

1. Bott J, Carroll MP, Conway JH, et al. Randomised controlled trial of nasal ventilation in acute ventilatory failure due to chronic obstructive airways disease. Lancet 1993; 341: 1555-1557.

2. Kramer N, Meyer J, Meharg J, Cece RD, Hill NS. Randomised, prospective trial of noninvasive positive pressure ventilation in acute respiratory failure. Am J Respir Crit Care Med 1995; 151: 1799-1806.

3. Brochard L, Mancebo J, Wysocki M, et al. Noninvasive ventilation for acute exacerbations of chronic obstructive pulmonary disease. $N$ Engl J Med 1995; 333: 817-822.

4. Celikel T, Sungur M, Ceyhan B, Karakurt S. Comparison of noninvasive positive pressure ventilation with standard medical therapy in hypercapnic acute respiratory failure. Chest 1998; 114: 1636-1642.

5. Plant PK, Owen JL, Elliott MW. Early use of non-invasive ventilation for acute exacerbations of chronic obstructive pulmonary disease on general respiratory wards. A multicenter randomised controlled trial. Lancet 2000; 355: 19311935.

6. International Consensus Conference in Intensive Care Medicine. Non invasive positive pressure ventilation in acute respiratory failure. Am J Respir Crit Care Med 2001; 163 : 283-291.

7. Baudouin S, Blumenthal S, Cooper B, et al. L. British Thoracic Society Guideline. Non-invasive ventilation in acute respiratory failure. Thorax 2002; 57:192-211.

8. Benahmou D, Girault C, Faure C, Portier F, Muir JF. Nasal mask ventilation in acute respiratory failure. Experience in elderly patients. Chest 1992; 102: 912-917.

9. Meduri GU, Fox RC, Abou-Shala N, Leeper KV, Wunderink RG. Noninvasive mechanical ventilation via face mask in patients with acute respiratory failure who refused endotracheal intubation. Crit Care Med 1994; 22: 1584-1590.

10. Carlucci A, Richard JC, Wysocki M, Lepage E, Brochard L. Noninvasive versus conventional mechanical ventilation. An epidemiological survey. Am J Respir Crit Care Med 2001; 163: 874-880.

11. Esteban A, Anzueto A, Frutos F, et al. Characteristics and outcomes in adult receiving mechanical ventilation. JAMA 2002; 287: 345-355.

12. Girault C, Briel A, Hellot MF, et al. Noninvasive mechanical ventilation in clinical practice: A 2-year experience in a medical intensive care unit. Crit Care Med 2003; 31: 552559.

13. Gorini M, Ginanni R, Villella G, Tozzi D, Augustynen A, Corrado A. Non-invasive negative and positive pressure ventilation in the treatment of acute on chronic respiratory failure. Intensive Care Med 2004; 70: 875-881.

14. Elliott MW. Improving the care for patients with acute severe respiratory diseases. Thorax 2003; 58: 285-288

15. European Respiratory Society Task Force on epidemiology of respiratory intermediate care in Europe. Respiratory intermediate care units: a European survey. Eur Respir $J$ 2002, 20: 1343-1350.

16. Conway JH, Hitchcock RA, Godfrey RC, Carroll MP. Nasal intermittent positive pressure ventilation in acute exacerbations of chronic obstructive pulmonary disease. A preliminary study. Respir Med 1993; 87: 387-394.

17. Confalonieri M, Aiolfi S, Gandola L, Scartabellati A, Della Porta, Parigi P. Severe exacerbations of chronic obstructive pulmonary disease treated with BiPAP by nasal mask. Respiration 1994; 61: 310-316.

18. Corbetta L, Ballerin L, Putinati S, Potena A. Efficacy of noninvasive positive pressure ventilation by facial and nasal mask in hypercapnic acute respiratory failure: experience in a respiratory ward under usual care. Monaldi Arch Chest Dis 1997; 52: 421-428.
19. Brown J, Jones D, Mikelsons C, Paul EA, Wedzicha JA. Using nasal intermittent positive pressure ventilation on a general respiratory ward. J R Coll Physicians Lond 1998; 32: 219-224.

20. Ferguson GT, Gilmartin M. $\mathrm{CO}_{2}$ rebreathing during BiPAP ventilatory assistance. Am J Respir Crit Care Med 1995; 151: 1126-1135.

21. Kelly BJ, Matthay HD. Prevalence and severity of neurological dysfunction in critically ill patients. Influence on need for continued mechanical ventilation. Chest 1993; 104: $1818-1824$

22. Seneff MG, Wagner DP, Wagner RP, Zimmerman JE Knaus WA. Hospital and 1-year survival of patients admitted to intensive care units with acute exacerbation of chronic obstructive pulmonary disease. JAMA 1995; 274: 1852-1857.

23. Charlson ME, Pompei P, Ales KL, MacKenzie CR. A new method of classifying prognostic co-morbidity in longitudinal studies: development and validation. J Chron Dis 1987; 40: 373-383.

24. Moretti M, Cilione C, Tampieri A, Fracchia C, Marchioni A, Nava S. Incidence and causes of non-invasive mechanical ventilation failure after initial success. Thorax 2000; 55: 819-825.

25. Knaus WA, Wagner DP, Draper EA, et al. The APACHE III prognostic system: risk prediction of hospital mortality for critically ill hospitalised adults. Chest 1991; 100: 16191936.

26. Katz MH. Multivariable analysis: a primer for readers of medical research. Ann Intern Med 2003; 138: 644-50.

27. Plant PK, Owen JL, Elliott MW. One year period prevalence study of respiratory acidosis in acute exacerbation of chronic obstructive pulmonary disease: implications for the provision of non-invasive ventilation and oxygen administration. Thorax 2000; 55: 550-554.

28. Appendini L, Patessio A, Zanaboni S, et al. Physiologic effects of positive end-expiratory pressure and mask pressure support during exacerbations of chronic obstructive pulmonary disease. Am J Respir Crit Care Med 1994; 149: 1069-1076.

29. Simonds AK. Nasal ventilation in progressive neuromuscular disease: experience in adults and adolescents. Monaldi Arch Chest Dis 2000; 55: 237-241.

30. Soo Hoo GW, Santiago S, Williams AJ. Nasal mechanical ventilation for hypercapnic respiratory failure in chronic obstructive pulmonary disease: determinants of success and failure. Crit Care Med 1994; 22: 1253-1261.

31. Ambrosino N, Foglio K, Rubini F, Clini E, Nava S, Vitacca $\mathrm{M}$. Non-invasive mechanical ventilation in acute respiratory failure due to chronic obstructive pulmonary disease: correlates for success. Thorax 1995; 50: 750-757.

32. Antonelli M, Conti G, Moro ML, et al. Predictors of noninvasive positive pressure ventilation in patients with acute hypoxemic respiratory failure: a multi-center study. Intensive Care Med 2001; 27: 1718-1728.

33. Domenighetti G, Gayer R, Gentilini R. Noninvasive pressure support ventilation in non-COPD patients with acute cardiogenic pulmonary edema and severe community-acquired pneumonia: acute effects and outcome. Intensive Care Med 2002; 28: 1226-1232.

34. Confalonieri M, Potena A, Carbone G, Della Porta R, Tolley EA, Meduri GU. Acute respiratory failure in patients with severe community-acquired pneumonia. A prospective randomised evaluation of non-invasive ventilation. Am J Respir Crit Care Med 1999; 160: 1585-1591.

35. Carlucci A, Delmastro M, Rubini F, Fracchia C, Nava S Changes in the practice of non-invasive ventilation in treating COPD patients over 8 years. Intensive Care Med 2003; 29: $419-25$ 